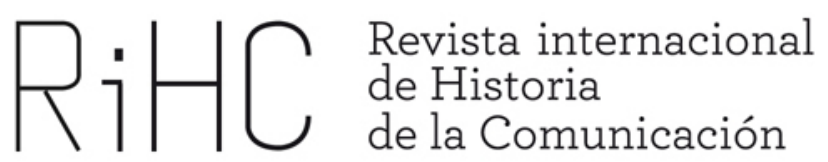

\title{
ANÁLISIS DE LA COBERTURA PERIODÍSTICA DE LA VANGUARDIA Y ABC, SOBRE LA CREACIÓN DE LAS FACULTADES DE CIENCIAS DE LA INFORMACIÓN EN ESPAÑA (CURSO ACADÉMICO 1971-1972)
}

DOI: http://dx.doi.org/10.12795/RiHC.2013.i01.09

Daniela Lazcano Peña

P. U. Católica de Valparaíso, Chile

daniela.lazcano@ucv.cl

Resumen: En los años 70 el periodismo y otras carreras del área de la Comunicación, vivieron una profunda transformación en su modo de enseñanza con la creación de las Facultades de Ciencias de la Información. Este trabajo analiza el modo en que este proceso se reflejó en parte de la prensa de la época, a través de la revisión de textos periodísticos publicados en La Vanguardia y $A B C$.

Palabras clave: Facultades de Comunicación en España, Enseñanza del Periodismo en España, análisis de prensa, historia de la comunicación

Abstract: In the 70's, the journalism -and other career related with Communicationexperimented a deep transformation in their teaching system: in that age, the journalism became in an university career. The present text is about this change, and about how this fact was told by the press, specifically on La Vanguardia and ABC. 
Análisis de la cobertura periodística de La Vanguardia y $A B C$, sobre la creación de las facultades de ciencias de la información en España (curso académico 1971-1972)

Keywords: Communication Schools in Spain, Journalism Education in Spain, News analysis, History of communication 


\section{Presentación: de la Escuela a la Universidad}

Durante el año académico 2012-2013, 36 universidades españolas formarán graduados en Periodismo ${ }^{1}$. Con esta cifra, los jóvenes interesados en el estudio y ejercicio profesional de los medios y las comunicaciones, cuentan una oferta de educación superior amplia y en sintonía con los procesos actuales de convergencia formativa internacional, tras su adaptación, en los últimos años, al Espacio Europeo de Educación Superior-EEES.

Esta cifra, además, refleja una enseñanza universitaria del Periodismo consolidada, y de rápido y constante crecimiento (ANECA, 2005: 100-104). Todo, a partir de los primeros años de la década de los 70, cuando la enseñanza del periodismo, y otras carreras vinculadas al campo de la comunicación, vivieron su incorporación al espacio formativo universitario.

Ocurrió en 1971. Ese año, y en el marco de la Ley General de Educación y Financiamiento de la Reforma Educativa, impulsada por el ministro de Educación y Ciencia de la época, José Luis Villar Palasí, se anunció la creación de las Facultades de Ciencias de la Información en las Universidades Complutense de Madrid y Autónoma de Barcelona.

Más allá del nuevo estatus disciplinario y académico que esta incorporación significó para el estudio del periodismo, el hecho puede comprenderse como parte de los procesos de transformación social y democrática que comenzaban a desarrollarse en España.

$Y$ es que a pesar de que la historia de la enseñanza del periodismo ya tenía antecedentes en el país -con las escuelas de El Debate o la Escuela Oficial de Periodismo, entre otros- los espacios formativos existentes se habían caracterizado por la fuerte orientación ideológica de parte de sus organismos rectores, por lo que la creación de las nuevas Facultades habría tenido como objetivo restar esta influencia y brindar mayor independencia a la formación periodística.

Este artículo analizará la presencia informativa -o noticiosa- de la creación de las Facultades de Ciencias de la Información. ¿Cómo se vivió el hecho? ¿Qué opiniones generó?, son algunas de las preguntas que buscarán respuesta a través de la revisión de los textos periodísticos publicados en este período por La Vanguardia y $A B C$, de

\footnotetext{
1 Información obtenida en el Registro de Universidades, Centros y Títulos (RUCT), de la Secretaría de Estado de Educación, Formación Profesional y Universidades, del Ministerio de Educación, Cultura y Deporte de España, a través de consulta on line realizada el 8 de diciembre de 2012, en el sitio https://www.educacion.gob.es/ruct/home.
} 
manera de identificar algunos de los argumentos y críticas con que se vivió, al menos en una parte de la prensa, la llegada del periodismo a las aulas universitarias.

\section{Un campo científico y el desarrollo de su enseñanza}

Hoy en día, el estudio de la Comunicación ha ganado -y gana- un espacio dentro de la comunidad académica y científica. Y es que más allá de los debates no resueltos sobre su condición y configuración epistemológica -donde se enmarcan preguntas sobre si es o no una disciplina, por ejemplo-, el campo cuenta ya con un devenir iniciado en los años 20 del siglo pasado.

Desde esa fecha son numerosos los modelos, enfoques, reflexiones e investigaciones que han tenido a la Comunicación como eje, derivando así en la configuración de un campo específico e institucionalizado. Es decir, en una comunidad académica activa y expandida en distintos países del mundo.

En el caso de España el estudio de la Comunicación es, hoy, un campo dinámico y en crecimiento. Sin embargo, éstas no siempre fueron sus características: mientras el interés científico y académico por el fenómeno comunicativo y mediático comenzaba a consolidarse en diferentes partes del mundo, su surgimiento en España se produjo de manera tardía. Los motivos: el régimen político surgido en la Guerra Civil, y la dictadura política que sufrió el país hasta 1975.

Así, el nacimiento de la disciplina de la Comunicación en España podría ubicarse en la última década del franquismo y a comienzos de la época democrático constitucional (García Jiménez, 2007: 155), proceso de nacimiento o emergencia ${ }^{2}$ (Martínez Nicolás, 2009) que tuvo como hito la creación de las Facultades de Ciencias de la Información de las universidades Autónoma de Barcelona y Complutense de Madrid. En este episodio, que significó la incorporación de los estudios de Periodismo al rango de carrera universitaria, se centra el presente texto ${ }^{3}$.

\footnotetext{
2 El autor Manuel Martínez Nicolás (2009) identifica tres fases en la configuración del campo de la Comunicación en España: 1) Emergencia: 1965 -1980; 2) Consolidación: 1980 - 1995; y 3) Desarrollo: 1995...

${ }^{3}$ Este artículo se enmarca en la investigación doctoral desarrollada por la autora sobre la configuración disciplinaria del campo de la comunicación, a través del análisis de su enseñanza en Facultades de Comunicación en España, y corresponde a su apartado de antecedentes históricos.
} 


\section{Enseñanza del periodismo en España}

Durante la última década de la dictadura franquista, el país comenzó a vivir una relativa liberalización política y económica marcada, entre otros factores, por el denominado "milagro español" y por la proclamación, en 1969, de Juan Carlos de Borbón, como sucesor -con el título de rey- de Francisco Franco.

En términos políticos y sociales, este proceso va de la mano con "la política de desarrollismo puesta en marcha por el franquismo desde finales de la década de los 50" (Martínez Nicolás, 2009: 3), y que tuvo como consecuencia la generación de las bases de una economía de mercado, el incipiente nacimiento de la sociedad de consumo, y la conversión de España como destino turístico del resto de Europa. "Esa política desarrollista irá generando las condiciones necesarias para el arraigo de una cultura de masas audiovisual en España, con la creación en 1956 de Radiotelevisión Española y el fuerte crecimiento del sector publicitario en la década de los 60 (en 1964 se funda la Escuela Oficial de Publicidad), impulsada por el turismo y el consumo interior" (Martínez Nicolás, 2009: 3). A estos hechos se sumaron, además, la "relajación" de la censura derivadas de las nueva Ley de Prensa de 1966, y la presión de los propios periodistas por la dignificación de la profesión, que se materializó en la creación del Grupo de Periodistas Democráticos, también en 1966 (Martínez Nicolás, 2009: 3), síntoma de un contexto en que "los profesionales de la información periodística requerían una formación que estuviese en consonancia con las repercusiones públicas de su actividad" (Real, 2009: 11).

De esta manera, "la dictadura empieza a relajar mínimamente el férreo control que había ejercido hasta entonces sobre el sistema cultural y comunicativo, y comienzan a darse a partir de entonces las condiciones sociales, académicas e intelectuales que permitirán el surgimiento de la investigación sobre comunicación como un campo disciplinar autónomo cultivado, a partir de ese momento, con criterios propiamente científicos y no aquéllos propagandísticos que dieran lugar a la entelequia bufonesca de una doctrina española de la información" (Martínez Nicolás, 2009: 3).

Dentro de este proceso, se suman y conjugan dos circunstancias que permitieron alcanzar la conversión universitaria de los estudios de periodismo: la nueva Ley General de Educación y Financiamiento de la Reforma Educativa, impulsada por el ministro de Educación y Ciencia de ese entonces, José Luis Villar Palasí ${ }^{4}$; y el cambio de

\footnotetext{
4 Esta La Ley General de Educación y Financiamiento de la Reforma Educativa comenzó su implementación en 1970, y estableció la enseñanza obligatoria hasta los 14 años con la Enseñanza General Básica. Tras ese período de formación, los estudiantes podían optar al Bachillerato Unificado Polivalente, o a la creada Formación Profesional. La última fase formativa sería la universitaria, donde se podrían cursar tres ciclos formativos: diplomados o carreras cortas (de tres años); licenciaturas (dos años más de especialización); y doctorados, último ciclo de mayor especialización, y orientados a quienes deseaban dedicarse a la docencia o investigación.
} 
gobierno de 1969, que ubicó a personas proclives al nuevo estatuto del periodismo-o cercanos-, en puesto claves de la administración. Entre ellos se contaba, por ejemplo, Emilio Romero, director del diario sindical Pueblo y designado como director de la Escuela Oficial del Periodismo por el recién nombrado ministro de Información y Turismo, Alfredo Sánchez Bella (Barrera: 2002). Para Romero, la llamada Ley Villar Palasí era "el último autobús que pasaba por delante de nosotros" para la incorporación universitaria del Periodismo, y trabajó para lograrlo: en mayo de 1970 presentó un plan ante la Federación Nacional de Asociaciones de la Prensa (Barrera: 2002).

En este contexto, y a pesar de que el tema no generaba unanimidad ni consenso como relata Barrera (2002) en 1968 un importante periodista de Pueblo había escrito que el periodismo no era materia universitaria, por lo que siempre sería una Escuela de formación profesional"- la Ley Villar Palasí incorporó entre sus reformas la enseñanza universitaria del periodismo y de otras carreras vinculadas al área de la Comunicación.

La nueva Ley planteó la creación en el curso académico 1971-1972 de las Facultades de Ciencias de la Información ${ }^{5}$ de las universidades Complutense de Madrid y Autónoma de Barcelona. Ese mismo año, el Instituto de Periodismo de Navarra, se transforma en Facultad de Ciencias de la Información de la Universidad de Navarra.

La enseñanza del Periodismo había llegado al mundo universitario. ${ }^{6}$

Dando una breve mirada hacia el pasado, la enseñanza del periodismo en España ya contaba con precedentes en el siglo XIX, con el curso organizado en la Universidad de Salamanca, en 1887, por el catedrático y periodista Fernando Arujo y Gómez; y en el XX, con la creación en 1926, por parte de Ángel Herrera Oria -quien más tarde sería Obispo de Málaga- de la Escuela de Periodismo del diario El Debate, que durante una década -hasta el inicio de la Guerra Civil en 1936- formó a un importante número de profesionales (Vigil y Vásquez 1987: 58).

\footnotetext{
${ }^{5}$ Para Mercedes Gordon Pérez (1991: 83) la denominación de "Facultad de Ciencias de la Información recoge la pluralidad de ciencias que se concentran en el estudio de la información y comunicación. La denominación para España fue fruto del esforzado trabajo del profesor Angel Benito, patrocinador de esta denominación por él defendida desde que en 1965 trocó el titulo que desde 1958 daba a los cursos de verano del Instituto de Periodismo de navarra, "Periodismo y Cuestiones de actualidad" que pasó a llamarse "Curso de Ciencias y Técnicas de la Información"

${ }^{6}$ Concretamente, este proceso se tradujo en que el título profesional de los estudios de periodismo, televisión, radio y publicidad, se obtendría tras cursar un plan de cinco años, estructurado en un primer ciclo de tres años que otorgaría el diplomado, más un segundo ciclo de dos años para la obtención de la licenciatura. Además, se consideraba la posibilidad de cursar un tercer ciclo, el doctorado, de mayor especialización y preparación para la docencia e investigación. En este artículo no se abordará la discusión generada sobre la incorporación de las otras carreras del campo de la comunicación a la universidad, sino que se centrará en específico sobre la enseñanza del periodismo.
} 
En 1941 la enseñanza del periodismo vuelve a tener un centro académico especializado: la Escuela Oficial de Periodismo (EOP), creada por el régimen franquista para la formación de profesionales para los medios de comunicación que respondieran a las necesidades, requerimientos e ideología del estado dictatorial (Moragas 1991/1981: 218-219). Con ella, el periodismo en España pasó de un modelo de enseñanza del periodismo empresa-escuela (con la Escuela de El Debate), a uno intervencionista totalitario (Pestano et al. 2011: 404-405)

Desde sus inicios, la Escuela Oficial de Periodismo, con una sede principal en Madrid, y una sección en Barcelona, fue un organismo dependiente del ministerio de Información y Turismo. En esa fecha, la Escuela Oficial era el único centro acreditado para otorgar el "carné" profesional, por lo que actuaba de examinador de los otros espacios que, por esos años, también contaban con cursos de enseñanza de periodismo.

Entre estos se contaba el Instituto de Periodismo del Estudio General de Navarra, que comenzó sus actividades académicas en 1958. Este espacio dependiente de la Iglesia católica y con fuerte influencia del Opus Dei, fue el primer centro con vocación universitaria, es decir, que combinaba el aspecto práctico con la teoría y reflexión.

A pesar de la existencia de estos centros formativos, para el desarrollo del campo de estudio de la comunicación su aportación "fue, por lo general, bien escasa; muy deficiente en la capacitación técnica para el trabajo periodístico, y prácticamente nula en la investigación de los fenómenos comunicativos. Por esta razón, entre otras, las escuelas de periodismo dificultaron el surgimiento de una comunidad científica sistemáticamente comprometida en el desarrollo de este campo disciplinar, que sólo comenzará a emerger cuando sus funciones sean asumidas por los nuevos centros" (Martínez Nicolás, 2006: 142), es decir, por las debutantes Facultades de Ciencias de la Información.

Así, una de sus mayores consecuencias fue la paulatina formación de una comunidad científica y el impulso del estudio y la investigación: "las Facultades de Ciencias de la Información han supuesto la entrada de nuestro país en el concierto internacional de las ciencias de la información y la comunicación. Las aportaciones científicas del profesorado español cubren todo el ancho campo de este supuesto científico y el número de revistas, obras colectivas, monografías y manuales es equiparable y aún superior en cantidad y calidad a lo que se publica en nuestro entorno europeo" (Benito 2002: 309).

Junto con este nuevo estatus científico, la acogida de su enseñanza en el espacio universitario significó, además, un paso en la independencia de la profesión, al cortar, 
aunque fuera en parte, con las influencias ideológicas directas existentes en el pasado. ${ }^{7}$

\section{Metodología}

Dado al interés y relevancia que la creación de las Facultades de Ciencias de la Información tuvo como punto de inicio de la investigación en Comunicación en España, este trabajo se ha desarrollado con el propósito de identificar y describir el modo en que parte de la prensa de la época informó sobre este suceso.

La aproximación a este objetivo se ha realizado a través de un análisis descriptivo de la cobertura de los temas relacionados con la creación de estas Facultades, en los diarios La Vanguardia y $A B C$.

Durante la época de este estudio, el diario de origen catalán La Vanguardia -o "La Vanguardia Española" como debió cambiar su cabecera por indicación del franquismo en aquellos años- tenía como director, desde 1969, a Horacio Sáenz Guerrero (Nogué y Barrera, 2006: 147), quien llevó el diario hacia un perfil más plural y de apoyo al cambio democrático, aunque siempre manteniendo su línea editorial liberalista, catalanista y monárquica.

EI ABC, por su parte, estaba en esos años bajo la dirección, por segunda vez, de Torcuato Luca de Tena (Olmos, 2002), nieto del fundador del diario, y fiel representante de su línea ideológica: conservador y monárquico. En esos años, $A B C$ contaba con dos ediciones: la de Madrid y la de Sevilla -existente desde 1929-, aunque para esta investigación se ha considerado sólo su edición madrileña.

Con la revisión de La Vanguardia y $A B C$, se ha buscado considerar la cobertura informativa de las dos ciudades donde se abrirían las primeras Facultades de Ciencias de la Información: Madrid y Barcelona.

La muestra se ha acotado a los textos periodísticos vinculados con la creación de las Facultades de Ciencias de la Información durante 1971, año en que se anuncia y pone en marcha la incorporación de la enseñanza del periodismo al ámbito universitario.

En base a esta delimitación temporal, se procedió a la constitución del corpus de análisis, conformado por la totalidad de noticias publicadas sobre esta temática, entre

\footnotetext{
${ }^{7}$ El Ministerio de Información y Turismo no renunció directamente al control de la enseñanza: estableció una Comisión Mixta entre los Ministerios de Información y Turismo y el de Educación, la cual fue la encargada de elaborar los primeros planes de estudio y controlar la primera contratación de profesores (Moragas 1991/1981: 269).
} 
el 1 de enero y el 31 de diciembre de 1971. El acceso a los textos se realizó a través de las hemerotecas digitales con que cuentan ambos medios. Tras este proceso se obtuvo un corpus compuesto por 85 textos. 38 de ellos correspondían a La Vanguardia, y 47 a la edición madrileña de $A B C$.

La Vanguardia Española

$A B C$ (edición Madrid)

Total

Tabla N¹: Corpus de noticias analizadas publicadas en La Vanguardia Española y ABC entre el 1 de enero y el 31 de diciembre de 1971, en relación a la creación de las Facultades de Cs de la Información.

Para el análisis de los textos, se trabajó con un instrumento de recolección de datos elaborado en base a la ficha de descripción de contenidos de periódicos utilizada por la Hemeroteca del Archivo Histórico de la Ciudad de Barcelona.

A ésta se han agregado dos campos: un número de identificación de la noticia analizada con respecto al total de informaciones publicadas sobre el tema durante el período analizado (el año 1971); y valoración o punto de vista sobre el tema analizado. Además, la descripción de título se ha complementado con información sobre epígrafe y bajada.

De la ficha original se eliminaron los campos referidos a descriptores topográficos y otros medios que hubieran publicado la noticia, por no considerarse pertinentes para el análisis realizado. La ficha se ha organizado en dos apartados: (1) de identificación formal de la noticia; y (2) de contenido. En la siguiente tabla se presenta un ejemplo de aplicación de esta ficha de análisis, a partir de una noticia publicada en La Vanguardia.

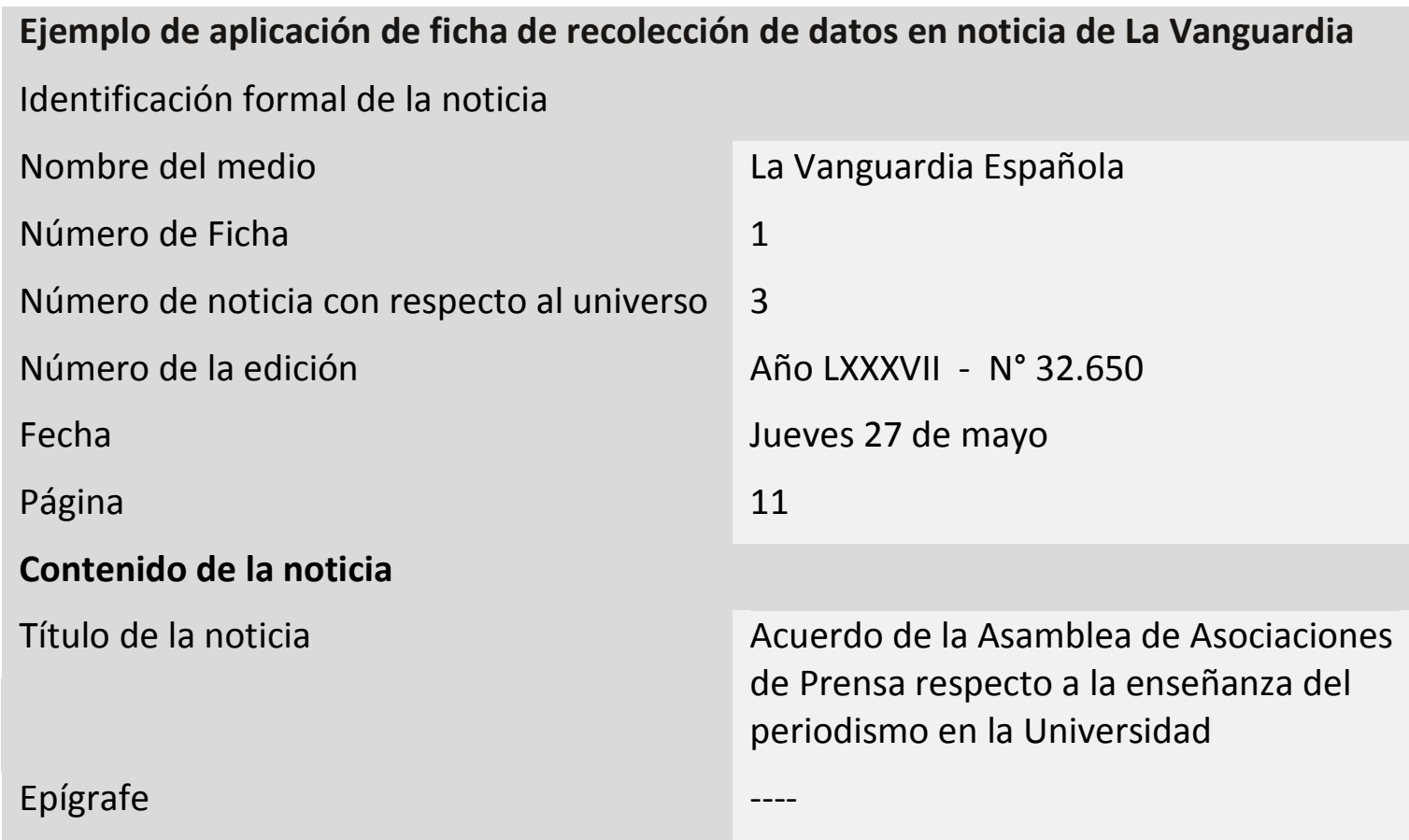




Bajada
Género
Autor
Resumen de la noticia
Materia principal
Materia secundaria
Personas citadas
Valoración o punto de vista sobre tema
analizado (Enseñanza universitaria del
periodismo)

Tercera jornada bilbaína

Informativo / crónica

Agencia Cifra

Resumen del último día de la Asamblea, considerando desde su agenda de actividades matinal y su punto de vista sobre el anuncio gubernamental de incorporar los estudios de periodismo de la Universidad

XXIX Reunión de la Federación Nacional de Asociaciones de la Prensa celebrada en la ciudad de Bilbao.

Creación de las Facultades de Ciencias de la Información y postura de la Federación

Lucio del Álamo, Presidente de la Federación Nacional de Asociaciones de la Prensa

Federación Nacional de Asociaciones de la Prensa

Integrantes de la Federación acuerdan "rogar" a los ministerios de Información y Turismo, y Educación y Ciencia, que en la regulación de la nueva Facultad de Ciencias de la Información, se tenga en cuenta:

1. Que para el ejercicio de la profesión periodística, desde la categoría de redactor, para los que salgan de la Facultad de Ciencias de la Información, sea exigible el título de licenciado en el departamento de periodismo de dicha facultad.

2. Que los periodistas actuales, debidamente titulados y los que obtengan su titulación, dentro de los planes en curso, de la Escuela Oficial de Periodismo, sean equiparados a todos los efectos a los licenciados que salgan de la Facultad de Ciencias de la Información, en su departamento de periodismo.

3. Que en el patronato de la nueva 
Facultad la representación corporativa de la profesión periodística sea lo suficientemente amplia y efectiva para intervenir en el control de la docencia.

4. Que los periodistas actualmente titulados y los que se titulen según los planes establecidos por la Escuela Oficial de Periodismo, puedan acceder a los estudios de doctorado en las mismas condiciones y circunstancias en que accederán los titulados que salgan de la futura facultad.

Tabla $\mathrm{N}^{\circ} 2$

Para acceder a un cuadro de síntesis del corpus total de trabajo, es decir, a las referencias de título, fecha, género, resumen, autor y personas e instituciones citadas de las 85 noticias trabajadas, se puede visitar el enlace $h t t p: / / d b . t t / R P 5 C y P r z$.

\section{Resultados y análisis}

El análisis de la información obtenida tras la aplicación de esta ficha a cada una de las 85 noticias, se ha orientado a la identificación de las principales tendencias y discrepancias presentes en la cobertura periodística de ambos medios.

En concreto, la atención se ha centrado en la lectura de cinco indicadores: (1) temporalidad de la cobertura; (2) tipología de los textos periodísticos publicados; (3) actores (personas e instituciones) citados; (4) principales materias abordadas; y (5) valoración o punto de vista presente en los textos periodísticos sobre el tema analizado.

\subsection{Temporalidad de la cobertura}

$\begin{array}{llll} & \text { La Vanguardia } & A B C & \text { Total mensual } \\ \text { Enero } & 0 & 0 & 0 \\ \text { Febrero } & 0 & 0 & 0 \\ \text { Marzo } & 0 & 0 & 0 \\ \text { Abril } & 1 & 0 & 1\end{array}$




$\begin{array}{llll}\text { Mayo } & 3 & 3 & 6 \\ \text { Junio } & 1 & 1 & 2 \\ \text { Julio } & 1 & 3 & 4 \\ \text { Agosto } & 3 & 6 & 9 \\ \text { Septiembre } & 5 & 10 & 15 \\ \text { Octubre } & 9 & 9 & 18 \\ \text { Noviembre } & 9 & 8 & 17 \\ \text { Diciembre } & 6 & 7 & 13 \\ \text { Total } & 38 & 47 & 85\end{array}$

Tabla N³: Distribución temporal de las noticias publicadas en La Vanguardia Española y ABC entre el 1 de enero y el 31 de diciembre de 1971, en relación a la creación de las Facultades de Ciencias de la Información.

Si bien "abril" fue el primer mes en que el anuncio sobre una próxima creación de las Facultades de Ciencias de la Información aparece en la prensa -y específicamente en La Vanguardia- ("A partir de octubre, Facultad de Ciencias de la Información en la Universidad Autónoma", 2 de abril, La Vanguardia), desde esa fecha y hasta diciembre, su presencia fue constante en los medios analizados, concentrando un auge entre los meses de septiembre y diciembre, como queda representado en el siguiente gráfico:

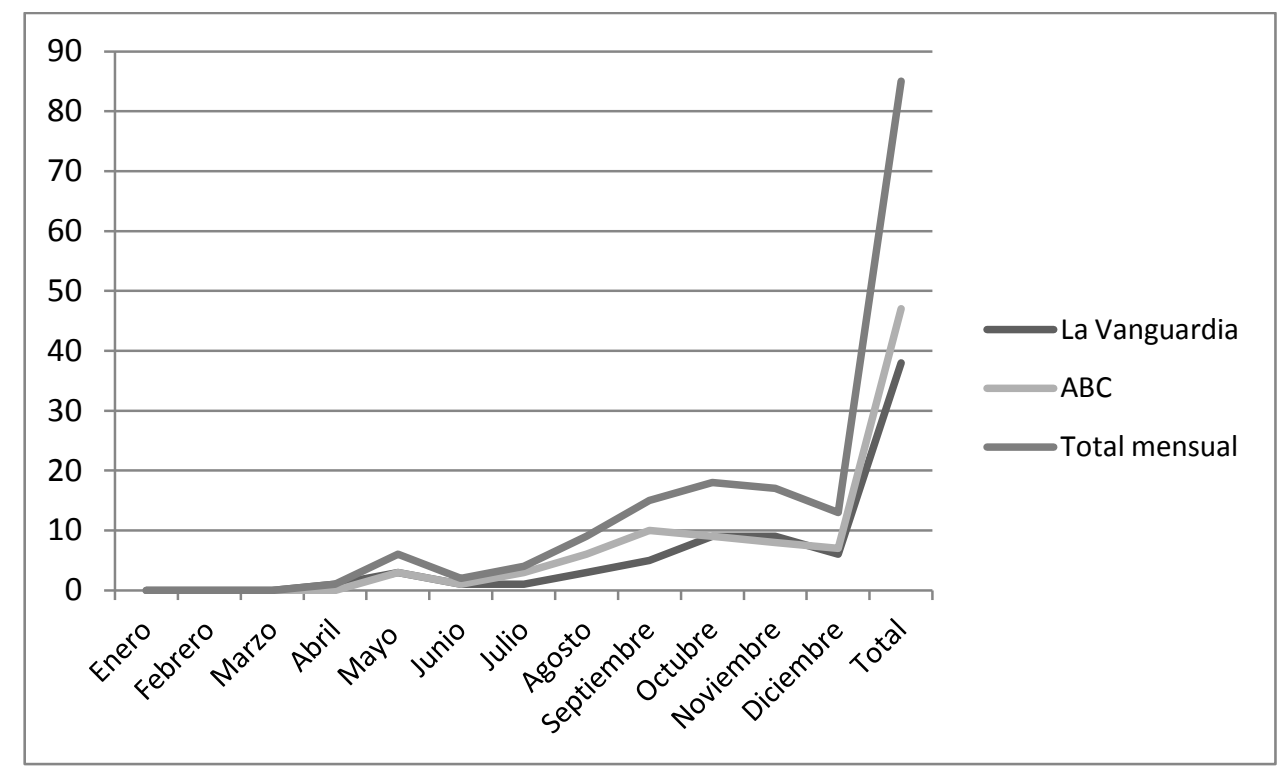

A mediados de octubre, se publica en el Boletín Oficial del Estado la creación de las Facultades de Ciencias de la Información de las Universidades Complutense de Madrid y Autónoma de Barcelona ("Por orden que insertó ayer el 'Boletín Oficial del Estado' del Ministerio de Educación y Ciencia, se crean las Facultades de Ciencias de la Información en las Universidades de Madrid (Complutense) y Autónoma de Barcelona", 17 de octubre, La Vanguardia; "Creación de las Facultades de Ciencias de la Información en 
las Universidad Complutense y Autónoma de Barcelona", 17 de octubre, mención en portada de $A B C)$.

Este hecho informativo marca el mes en que se intensifican las informaciones con antecedentes administrativos de su puesta en marcha (matrícula, planes de estudio, reglamentos, entre otras temáticas), las que deben convivir con algunas posiciones críticas y cuestionamientos, en especial, por parte de asociaciones profesionales de la prensa.

Esta tendencia -noticias informativas en paralelo con textos que analizan con preocupación la puesta en marcha de esta transformación en la enseñanza del periodismo- se mantiene hasta finales de ese año ("En la Universidad Complutense. Tomó posesión el decano-comisario de la Facultad de Ciencias de la Información", 19 de noviembre, ABC; "Preocupación entre los actuales profesionales de la información", 1 de diciembre, La Vanguardia).

\subsection{Tipología de textos periodísticos}

$\begin{array}{llll} & \text { La Vanguardia } & \text { ABC } & \text { Total por género } \\ \text { Breves } & 16 & 29 & 45 \\ \text { Crónicas } & 17 & 16 & 33 \\ \text { Cartas lectores } & 2 & 2 & 4 \\ \text { Columnas de opinión } & 2 & 0 & 2 \\ \text { Caricatura } & 1 & 0 & 1 \\ \text { Total } & 38 & 47 & 85\end{array}$

Tabla N4: Tipología de los textos periodísticos publicados en La Vanguardia Española y ABC entre el 1 de enero y el 31 de diciembre de 1971, en relación a la creación de las Facultades de Ciencias de la Información.

Entre las noticias analizadas la mayor presencia se concentró en textos informativos breves, de uno o dos párrafos, que daban cuenta de hechos puntuales, como convocatorias a los estudiantes, nombramiento de autoridades o inicio de clases. En algunos casos, estos "breves" se incluían como materia secundaria dentro de informaciones sobre otros temas vinculados a personas o instituciones del mundo del periodismo o la educación.

En cuanto a géneros periodísticos, lo más utilizado fue la aproximación informativa. A ésta se sumaron algunos espacios de opinión, como cartas al director $y$, en el caso de La Vanguardia, dos columnas de opinión y una viñeta de humor. 
Si bien no se ha considerado como un indicador independiente, vale la pena mencionar que en el caso de $A B C$, tres de las informaciones aparecieron como menciones en la portada del diario ("Facultad de Ciencias de la Información", 14 de agosto; "Creación de Facultades de Ciencias de la Información en las Universidades Complutense de Madrid y Autónoma de Barcelona", 1 de septiembre y 17 de octubre -mismo titular-).

Por otro lado, y en cuanto a la autoría de los textos, en ambos medios la gran mayoría provenía de agencias informativas (como Europa Press y Cifra), corresponsales del diario, o la propia redacción del medio.

\subsection{Actores de las informaciones}

$\begin{array}{lll}\text { Actores (personas e instituciones) } & \text { La Vanguardia } & \text { ABC } \\ \text { Universidades } & 28 & 33 \\ \text { Organizaciones gremiales } & 17 & 13 \\ \text { Gobierno } & 8 & 6 \\ \text { Centros de formación de Periodismo } & 8 & 4 \\ \text { Iglesia } & 7 & 5 \\ \text { Universidades } & 28 & 33\end{array}$

Tabla $N^{\circ} 5$ : Actores (personas e instituciones) citados en los textos periodísticos publicados en La Vanguardia Española y ABC entre el 1 de enero y el 31 de diciembre de 1971, en relación a la creación de las Facultades de Ciencias de la Información.

Del análisis global se identifican cinco grandes grupos de actores que, ya sea a través de referencia a instituciones o personas concretas, predominan como protagonistas del paso del periodismo a la universidad.

Entre estos, destacan las Universidades, tanto aquellas en que funcionarán las nuevas Facultades (UCM y UAB), como aquellas que manifiestan su interés por contar con una (Universidad de Salamanca, Universidad Autónoma de Bilbao, Universidad Hisplanense).

Otros actores protagónicos son las organizaciones gremiales de profesionales de la prensa, como la Federación Nacional de Asociaciones de Prensa, o las Asociaciones de Prensa de Madrid y Barcelona, entidades que tuvieron presencia en ambos medios a través de la expresión de sus opiniones sobre la creación de las Facultades de Ciencias de la Información, como se desarrollará en un apartado posterior. 
A estos se suman, también, el Gobierno, entidad encargada de la voz oficial sobre la creación de las Facultades en el marco de la Ley General de Educación; y los Centros de Formación de Periodismo, como la Escuela Oficial de Periodismo y la Escuela de Periodismo de la Iglesia, en especial por informaciones que daban cuenta de su futuro tras el paso de la enseñanza del periodismo a la universidad.

Finalmente, otro de los actores que marcó presencia fue la Iglesia, tanto a través de su ya nombrada Escuela de Periodismo de la Iglesia, como por la manifestación explícita de los organismos eclesiásticos de su voluntad de contar con una enseñanza universitaria del periodismo, a través de, por ejemplo, la Pontificia Universidad de Salamanca.

En el siguiente gráfico se explicita la presencia en ambos diarios de los actores genéricos ya reseñados.

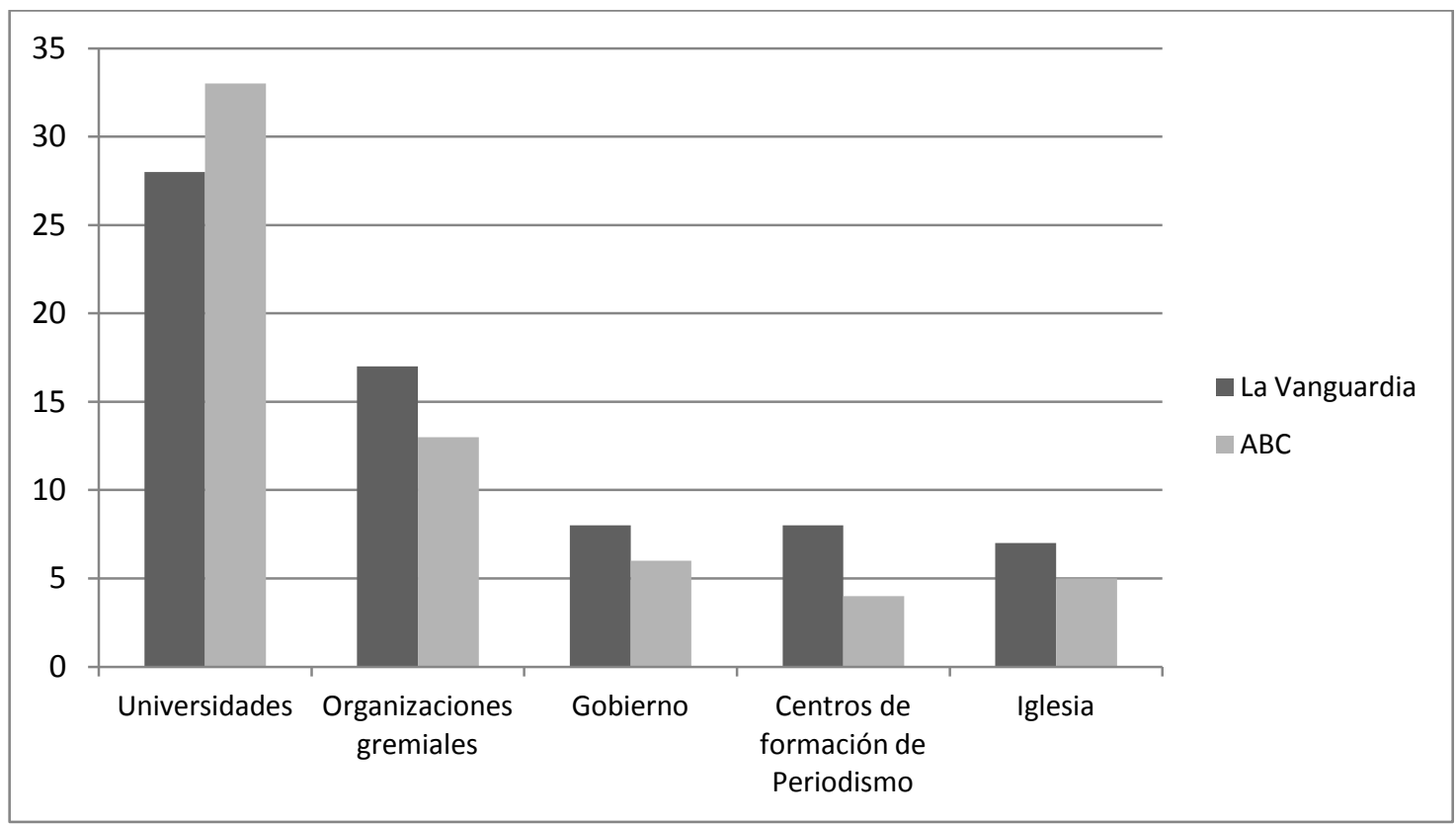

Para complementar la entrega global de resultados, en la tabla $n^{\circ} 6$ se entrega el detalle las personas e instituciones que, en específico, forman parte de los cinco actores genéricos codificados.

\begin{tabular}{|c|c|c|c|c|c|}
\hline & & La Vanguar & & $A B C$ & \\
\hline $\begin{array}{l}\text { Actores } \\
\text { principales }\end{array}$ & & $\begin{array}{l}\text { Menciones } \\
\text { por ítem }\end{array}$ & $\begin{array}{l}\text { Total } \\
\text { global ítem }\end{array}$ & $\begin{array}{l}\text { Menciones } \\
\text { por ítem }\end{array}$ & $\begin{array}{l}\text { Total } \\
\text { global } \\
\text { por ítem }\end{array}$ \\
\hline Universidades & & & 28 & & 33 \\
\hline Universidad & UCM (mención institucional) & 6 & 12 & 11 & 17 \\
\hline $\begin{array}{l}\text { Complutense de } \\
\text { Madrid }\end{array}$ & Rector UCM, Botella Lluisá & 1 & & 4 & \\
\hline & $\begin{array}{l}\text { Adolfo Muñoz Alonso, } \\
\text { decano Fac de Cs de la Inf } \\
\text { UCM }\end{array}$ & 5 & & 2 & \\
\hline
\end{tabular}




\begin{tabular}{|c|c|c|c|c|c|}
\hline \multirow{3}{*}{$\begin{array}{l}\text { Universidad } \\
\text { Autónoma de } \\
\text { Barcelona }\end{array}$} & UAB (mención institucional) & 8 & 11 & 4 & \multirow[t]{3}{*}{11} \\
\hline & $\begin{array}{l}\text { Rector UAB, Vicente Villar } \\
\text { Palasí }\end{array}$ & 2 & & 4 & \\
\hline & $\begin{array}{l}\text { Martín de Riquer, posible } \\
\text { decano de la Fac de Cs de la } \\
\text { Inf UAB }\end{array}$ & 1 & & 3 & \\
\hline \multirow{2}{*}{$\begin{array}{l}\text { Pontificia } \\
\text { Universidad de } \\
\text { Salamanca }\end{array}$} & $\begin{array}{l}\text { U de Salamanca (mención } \\
\text { institucional) }\end{array}$ & 3 & 3 & 0 & \multirow[t]{2}{*}{1} \\
\hline & $\begin{array}{l}\text { Gran Canciller U de } \\
\text { Salamanca }\end{array}$ & 0 & & 1 & \\
\hline $\begin{array}{l}\text { Universidad de } \\
\text { Navarra }\end{array}$ & $\begin{array}{l}\text { Angel Benito, director } \\
\text { Instituto de Periodismo de la } \\
\text { U. de Navarra. }\end{array}$ & 2 & 2 & 2 & 2 \\
\hline \multicolumn{2}{|c|}{ Universidad Hisplanense } & 0 & 0 & 1 & 1 \\
\hline \multicolumn{2}{|c|}{ Universidad Autónoma de Bilbao } & 0 & 0 & 1 & 1 \\
\hline \multicolumn{2}{|c|}{ Organizaciones gremiales } & & 17 & & 13 \\
\hline \multirow{2}{*}{$\begin{array}{l}\text { Federación de } \\
\text { Asociaciones de la } \\
\text { Prensa de España }\end{array}$} & $\begin{array}{l}\text { Federación (mención } \\
\text { institucional) }\end{array}$ & 4 & 10 & 1 & \multirow[t]{2}{*}{7} \\
\hline & $\begin{array}{l}\text { Lucio del Álamo, Presidente } \\
\text { de la Federación }\end{array}$ & 6 & & 2 & \\
\hline \multicolumn{2}{|c|}{ Asociación de Prensa de Madrid } & 1 & 1 & 3 & 3 \\
\hline \multicolumn{2}{|c|}{ Asociación de Prensa de Barcelona } & 1 & 1 & 1 & 1 \\
\hline \multirow{2}{*}{$\begin{array}{l}\text { Agrupación } \\
\text { Sindical de Radio y } \\
\text { Televisión }\end{array}$} & $\begin{array}{l}\text { Agrupación Sindical } \\
\text { (mención institucional) }\end{array}$ & 1 & 3 & 0 & \multirow[t]{2}{*}{2} \\
\hline & Manuel Aznar, presidente & 2 & & 2 & \\
\hline $\begin{array}{l}\text { Sindicato Nacional } \\
\text { de Prensa, Radio, } \\
\text { Televisión y } \\
\text { Publicidad }\end{array}$ & $\begin{array}{l}\text { Presidente, don Antonio } \\
\text { Castro Villacañas, }\end{array}$ & 1 & 1 & 0 & 0 \\
\hline $\begin{array}{l}\text { Dirección general } \\
\text { de Radiodifusión y } \\
\text { Televisión }\end{array}$ & Director, don Adolfo Suárez & 1 & 1 & 0 & 0 \\
\hline \multicolumn{2}{|l|}{ Gobierno } & & 8 & & 6 \\
\hline \multicolumn{2}{|c|}{ Ministro de Educación y Ciencia } & & 4 & & 1 \\
\hline \multicolumn{2}{|c|}{$\begin{array}{l}\text { Alfredo Sánchez Bella, ministro de información y } \\
\text { turismo }\end{array}$} & & 2 & & 2 \\
\hline \multirow[t]{3}{*}{ Otras autoridades } & Jefe de Estado & 1 & 2 & 1 & 3 \\
\hline & Consejo de Ministros & 1 & & 1 & \\
\hline & Autoridades de gobierno & 0 & & 1 & \\
\hline \multicolumn{2}{|c|}{ Centros de formación de Periodismo } & & 8 & & 4 \\
\hline \multirow{2}{*}{$\begin{array}{l}\text { Escuela Oficial de } \\
\text { Periodismo }\end{array}$} & EOP (mención institucional) & 3 & 6 & 1 & 3 \\
\hline & Emilio Romero, director EOP & 3 & & 2 & \\
\hline Escuela de & Escuela (mención & 1 & 2 & 0 & 1 \\
\hline
\end{tabular}




\begin{tabular}{|c|c|c|c|c|c|}
\hline \multirow{2}{*}{$\begin{array}{l}\text { Periodismo de la } \\
\text { Iglesia }^{8}\end{array}$} & \multicolumn{5}{|l|}{ institucional) } \\
\hline & $\begin{array}{l}\text { Alejandro Fernández Pombo, } \\
\text { Director }\end{array}$ & 1 & & 1 & \\
\hline \multicolumn{2}{|l|}{ Iglesia } & & 7 & & 5 \\
\hline \multicolumn{2}{|l|}{ Arzobispado } & 1 & 1 & 0 & 0 \\
\hline \multicolumn{2}{|c|}{$\begin{array}{l}\text { Comisión Episcopal de Medios de Comunicación } \\
\text { Social }\end{array}$} & 4 & 4 & 2 & 2 \\
\hline \multicolumn{2}{|c|}{ Arzobispo de Zaragoza } & 0 & 0 & 1 & 1 \\
\hline \multicolumn{2}{|c|}{ Conferencia Episcopal } & 0 & 0 & 1 & 1 \\
\hline \multirow{2}{*}{$\begin{array}{l}\text { Escuela de } \\
\text { Periodismo de la } \\
\text { Iglesia }\end{array}$} & $\begin{array}{l}\text { Escuela (mención } \\
\text { institucional) }\end{array}$ & 1 & 2 & 0 & 1 \\
\hline & $\begin{array}{l}\text { Alejandro Fernández Pombo, } \\
\text { Director }\end{array}$ & 1 & 1 & 1 & 0 \\
\hline
\end{tabular}

Tabla N6: Detalle de las personas e instituciones citados en los textos periodísticos publicados en La Vanguardia Española y $A B C$ entre el 1 de enero y el 31 de diciembre de 1971, en relación a la creación de las Facultades de Ciencias de la Información

Contrastando la revisión de estos textos informativos con bibliografía que relata la historia de este proceso, resulta interesante que las protestas y huelgas que habrían realizado los alumnos de la Escuela Oficial de Periodismo (Vigil y Vázquez 1987: 148), como una manera de demostrar su oposición ante la creación de las Facultades de Ciencias de la Información -y el consecuente cierre de la EOP- y que habrían tenido lugar durante el período estudiado, no tiene ninguna aparición en los medios analizados.

\subsection{Principales temáticas abordadas}

\begin{tabular}{lll} 
Temas principales & La Vanguardia & ABC \\
$\begin{array}{l}\text { Creación y funcionamiento nuevas Facultades de Cs } \\
\text { de la Información }\end{array}$ & 18 & 33 \\
$\begin{array}{l}\text { Posición de organismos profesionales ante creación } \\
\text { de Facultades de Cs de la Información) }\end{array}$ & 6 & 5 \\
$\begin{array}{l}\text { Situación de antiguas Escuelas } \\
\begin{array}{l}\text { Reflexiones sobre paso de Periodismo a Universidad } \\
\text { (incluidas ironías) }\end{array}\end{array}$ & 5 & 2 \\
$\begin{array}{l}\text { Posición gubernamental ante preocupación de } \\
\text { periodistas sobre creación de Facultades de Cs de la }\end{array}$ & 1 & 1 \\
\hline
\end{tabular}

\footnotetext{
${ }^{8}$ Los datos relativos a la Escuela de Periodismo de la Iglesia se han considerado parte de dos categorías de actores genéricos: Centros de Formación de Periodismo, e Iglesia.
} 
Información

Otras posibles Facultades de Cs de la Información

Tabla N7: Temáticas principales de los textos periodísticos publicados en La Vanguardia Española y ABC entre el 1 de enero y el 31 de diciembre de 1971, en relación a la creación de las Facultades de Ciencias de la Información.

Las 85 noticias estudiadas pueden ser agrupadas en seis áreas temáticas. La primera de ellas, y de mayor presencia, es la referida a la creación y funcionamiento de las nuevas Facultades: en ambos medios comprende más de la mitad de los textos publicados, y abarca desde los primeros anuncios de la creación de las Facultades, hasta el nombramiento de autoridades, reglamentos, procedimientos de matrícula o fecha de inicio de clases.

Como se mencionó, en La Vanguardia, y ya desde la noticia publicada el 2 de abril, se anuncia a la Universidad Autónoma de Barcelona como una de las instituciones en que se ubicaría una de las nuevas. El $A B C$, en tanto, pospone esta confirmación hasta agosto ("Tres nuevas facultades en la Autónoma de Barcelona", 28 de agosto).

Antes de esa fecha, en el medio madrileño se dio tribuna a informaciones que planteaban el interés de otras universidades por contar con una de estas nuevas Facultades ("La universidad Hispalense solicita una Facultad de Ciencias de la Información", 9 de septiembre, "Estos días pasados han tenido lugar reuniones de varios señores obispos de la Comisión Episcopal de Medios de Comunicación Social, presididos por el señor arzobispo de Zaragoza y miembros de la Pontificia Universidad de Salamanca (...) Se trató en estas conversaciones de la construcción de una Facultad de Ciencias de la Información en la referida Universidad Pontificia", 1 de octubre; "El futuro tiene que ser prometedor (...) También ha pedido la Universidad Autónoma de Bilbao una Facultad de Ciencias de la Información", 6 de octubre).

También en el $A B C$, se abordó una particularidad con respecto al funcionamiento de la Facultad de Ciencias de la Información de la Complutense: la construcción de su sede, la que estuvo impregnada por problemas urbanísticos, haciendo peligrar su apertura (“El área metropolitana eleva su preocupación al gobierno por el exceso de edificaciones en la ciudad universitaria. Este punto, además de otros motivos, ha influido en el informe desfavorable para un circuito experimental de sodio y la Facultad de Ciencias de la Información", 20 de noviembre).

En un segundo nivel de protagonismo se ubica la posición de los organismos profesionales. Esta temática se inicia en el mes de mayo, con la inauguración de la Asamblea de las Asociaciones de Prensa en Bilbao, ocasión en que se da a conocer, por parte del Gobierno, de la futura creación de las Facultades de Ciencias de la Información. 
Como resultado de la asamblea, se elabora un documento que plantea, por primera vez dentro de las noticias analizadas, la preocupación de los actuales periodistas por el paso del periodismo a la universidad. Esta temática se mantuvo a lo largo de todo el año, potenciada por la adhesión de otras Asociaciones de la Prensa, como la Madrid y Barcelona, a los planteamientos de la Federación ("La Federación de Asociaciones de Prensa pide que se aplace la puesta en marcha de las Facultades de Ciencias de la Información", 26 de octubre, La Vanguardia; "Peticiones de los profesionales del periodismo en relación con su titulación. Fueron aprobadas anoche en la Junta general de la Asociación de Prensa de Madrid", 27 de octubre, ABC).

Aunque con una presencia menor, otras temáticas abordadas son la situación de las antiguas escuelas de periodismo; el ya mencionado interés de otros centros universitarios por contar con enseñanza universitaria del periodismo; y la posición gubernamental frente a este proceso.

Por último, una mención especial a una temática que se hizo presente especialmente en La Vanguardia: la reflexión global, a través de artículos de opinión, sobre el significado del paso del periodismo a la universidad. Este tema, y si bien es escaso en términos cuantitativos, sí resulta interesante en lo cualitativo, pues es explícito en manifestar un punto de vista crítico sobre la enseñanza universitaria del periodismo.

En el siguiente gráfico se explicita la presencia en ambos diarios de las principales temáticas abordadas:

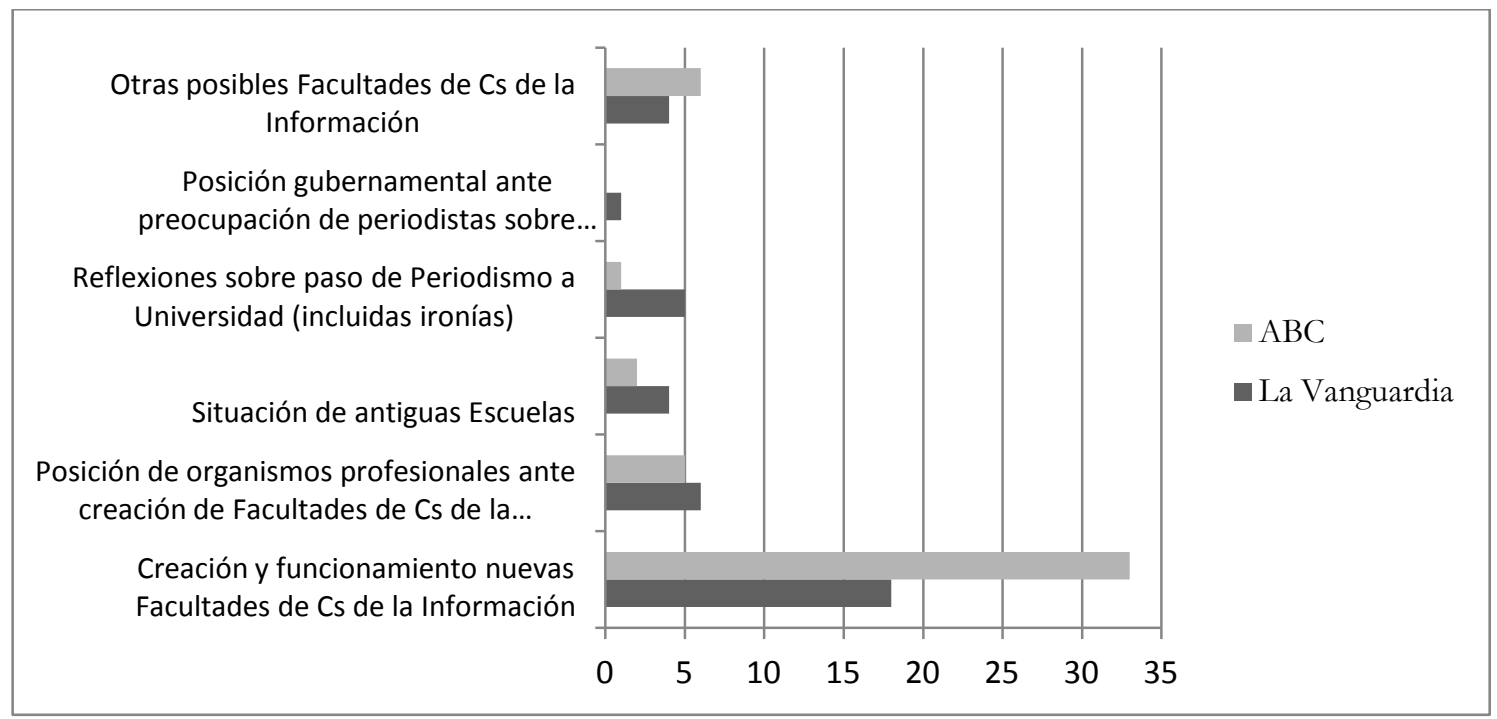




\subsection{Valoración o punto de vista}

El último de los indicadores considerados en el análisis, ha sido denominado como "Valoración o punto de vista", es decir, las opiniones o perspectivas que son posibles de desprender, como tendencias, en los textos analizados.

Como queda reflejado en la tabla $n^{\circ} 8$, las 85 noticias revisadas pueden ser agrupadas en cuatros valoraciones generales: Neutra-informativa; preocupación de organismos gremiales; valoración positiva; críticas e incertidumbres.

$\begin{array}{lll}\text { Temas principales } & \text { La Vanguardia } & \text { ABC } \\ \text { Neutra - Informativa } & 17 & 31 \\ \text { Preocupación organismos gremiales } & 9 & 9 \\ \text { Valoración positiva } & 5 & 4 \\ \text { Críticas e incertidumbres } & 7 & 1\end{array}$

Tabla Nº: Valoración o punto de vista de los textos periodísticos publicados en La Vanguardia Española y $A B C$ entre el 1 de enero y el 31 de diciembre de 1971, en relación a la creación de las Facultades de Ciencias de la Información

El primer concepto, neutra-informativa, presente de manera predominante, es coherente con el gran protagonismo que tienen los textos relacionados con la temática de creación y funcionamiento de las Facultades de Ciencias de la Información, presentados en su gran mayoría en formato de breves informativos, como se indicaba en el punto 5.2.

Entre las otras valoraciones, destaca la denominada preocupación de organismos gremiales. Esta perspectiva se manifestó de manera constante a largo de 1971, y fue expresado por diferentes organismos gremiales de profesionales de la prensa.

A grandes rasgos, se presenta como mirada positiva al hecho global de la consideración universitaria del periodismo, pero con una alta preocupación por la situación en que quedarían quienes en ese momento ya estaban ejerciendo la profesión. En este sentido, las publicaciones enfatizan tanto en el deseo de ser parte del proceso de incorporación del periodismo a la universidad, como en el establecimiento de garantías para los periodistas.

Así, por ejemplo, el 27 de mayo, La Vanguardia informaba de las conclusiones de la XXIX Asamblea General de la Federación Nacional de Asociaciones de Prensa:

"Tras numerosas deliberaciones se tomó el acuerdo de rogar conjuntamente a los ministros de Información y Turismo y Educación y Ciencia que en la regulación de esta nueva facultad se tengan en cuenta las siguientes aspiraciones de la asamblea: $1^{\circ}$ Que para el ejercicio de la profesión periodística, desde la categoría 
de redactor, para los que salgan de la Facultad de Ciencias de la Información, sea exigible el título de licenciado en el departamento de periodismo de dicha facultad. $2^{\circ}$ Que los periodistas actuales debidamente titulados y los que obtengan su titulación, dentro de los planes en curso, de la Escuela Oficial de Periodismo, sean equiparados a todos los efectos a los Licenciados que salgan de la Facultad de Ciencias de la Información en su departamento de periodismo. $3^{\circ}$ Que en el patronato de la nueva facultad la representación corporativa de la profesión periodística sea lo suficientemente amplia y efectiva para intervenir en el control de la docencia. $4^{\circ}$ Que los periodistas actualmente titulados y los que se titulen en alguno de los planes establecidos por la Escuela Oficial de Periodismo, puedan acceder a los estudios del doctorado en las mismas condiciones y circunstancias en que accederán los titulados que salgan de la futura facultad".

A lo largo del año, otras Asociaciones de Prensa del país se fueron sumando a estas peticiones, y adicionando nuevos enfoques, como la postergación de su puesta en marcha ("Que se planifiquen los estudios de periodismo sin precipitación. Las Asociaciones de Prensa piden también el aplazamiento de la puesta en marcha de las nuevas Facultades", 26 de octubre, $\mathrm{ABC}$ ).

Estas preocupaciones, en alguna ocasión, fueron asumidas por el propio Gobierno, valorando el traspaso del periodismo a la universidad ("El periodismo encontrará en la nueva Facultad de Ciencias de la Información el clima apropiado para desarrollarse en profundidad y rigor", declaraciones del Ministro de Información y Turismo, 8 de junio, $A B C$ ), y aplacando los temores que este proceso generaba en los ya profesionales ("No habrá, mientras yo esté en el Ministerio, ningún peligro de los que han preocupado en estos días en algunos ambientes periodísticos", declaraciones del Ministro de Información y Turismo, 8 de junio, La Vanguardia).

También como parte de las miradas positivas, aunque no de gran presencia mediática, se cuentan las declaraciones que desde el mundo universitario valoraban el cambio como un avance académico, científico y profesional ("La aparición de las Facultades de ciencias de la información señala en España la decisiva comprensión del hecho informativo como un supuesto científico (...) indican el reconocimiento de las responsabilidades sociales de los profesionales de la información", declaraciones de Ángel Benito, director del Instituto de Periodismo de la U. de Navarra, 18 de noviembre, $A B C)$.

Finalmente, como críticas e incertidumbres, se ubican aquellos textos que cuestionan la puesta en marcha de las Facultades, ya sea por temas administrativos o, como ya se ha indicado, por considerarse que el proceso se he realizado con demasiada celeridad. En esta categoría resultan interesantes los artículos de opinión que se publicaron en La Vanguardia que, como se planteó anteriormente, aunque escasos, son relevantes por su mirada crítica. 
En este contexto, el 5 de noviembre, el reconocido periodista Augusto Assia, en su sección de Cartas el Director de La Vanguardia, se pregunta, no sin un dejo de ironía “¿Bastará con que un periodista tenga título de la Universidad y otro no para que todos los españoles se lancen a devorar lo que escribe el universitario, y a darle crédito, mientras le vuelven la espalda y desacreditan cuanto pueda escribir el otro?".

Ese mismo día, el caricaturista Perich, parece subrayar las palabras de Assia con la imagen de alguna persona de cargo relevante (un ejecutivo, empresario o alto funcionario de gobierno) que, sentado tras su escritorio, advierte a su secretaria "No se olvide señorita Montse... iSólo concedo entrevistas a doctores en periodismo!" (La Vanguardia, 5 de noviembre 1971).

\section{Conclusiones}

Finalizado el análisis descriptivo, es posible plantear algunas ideas globales, como conclusiones de este trabajo.

Lo primero es indicar que, salvo leves matices, tanto $A B C$ como La Vanguardia muestran claras similitudes en el modo de informar sobre el proceso de traspaso de la enseñanza del periodismo a la universidad. La mayor discrepancia se produce, quizás, en el tono crítico e irónico que, en contadas ocasiones, se presentó en las páginas del diario catalán.

Entre los otros resultados, es posible destacar algunas tendencias comunes:

- Presencia constante: el tema contó con cobertura durante todo el año, con un auge de tres meses, siendo parte, incluso, de informaciones que no lo consideraban como noticia principal.

- Camino a la democracia: desde el inicio de este texto se han recogido argumentos que plantean que el paso del periodismo a la universidad no puede sino entenderse dentro de un contexto de avance hacia la transición democrática.

Esta perspectiva, si bien no se presenta de manera general en las noticias analizadas, sí cuenta con algunas referencias directas. Una, por parte del ministro de Información y Turismo quien, al anunciar la futura creación de las Facultades, lo valora como parte de los avances que en materia de libertad social y de prensa se han generado en España gracias al régimen franquista:

"Los diarios españoles han ido perfeccionándose, y a efectos de su actuación, cara a la opinión pública, han conseguido un sistema 
convencional, abierto a la crítica y rico en contrastes, con ideas y opiniones dispares muchas veces, pero, en general, con un acuerdo tácito de defender y de respetar lo que ha sido ya la labor de dos generaciones de españoles (...) La interpretación sociedad-Estado en la España presente es una realidad forjada por Franco, y culminada institucionalmente el 22 de julio de 1969 (...) El Ministerio de Información y Turismo está al servicio de este nuevo horizonte y en apoyo de todas las ideas positivas que puedan ayudar a los españoles en esta etapa de su desarrollo político, social y económico" (8 de junio, $A B C)$.

Otra referencia, orientada a destacar la libertad que al ejercicio del periodismo ofrecerá este cambio, se puede encontrar en las declaraciones del académico Ángel Benito, en la apertura del curso del Colegio Mayor Aralar:

"Los periodistas están necesitados cada día de una mayor preparación. La aparición de las Facultades señala el propósito de dotar de autonomía e independencia a los centros académicos destinados a capacitar a unos profesionales en los que la sociedad ha depositado la gestión delicada de los medios de información" (18 de noviembre, $\mathrm{ABC}$ ).

- Protagonismo de las organizaciones profesionales: a pesar de la presencia de actores como el gobierno, las universidades o las escuelas de periodismo, el claro protagonismo lo tuvieron las organizaciones de prensa, es decir, las entidades gremiales que representaban a los periodistas ya titulados, quienes fueron parte constante de la cobertura, a través de las manifestaciones de sus puntos de vista.

De lo que se puede desprender de los textos periodísticos analizados, resulta interesante que este protagonismo se habría agotado en el plano del discurso, es decir, habrían manifestado sus aprensiones y deseos de ser parte de este proceso, pero no habrían logrado una participación real, lo que se infiere por la mantención y aumento de sus críticas y objeciones, en el período analizado.

- Profesionales de primera y de segunda: parte importante de las argumentaciones presentadas por la Federación Nacional de Asociaciones de Prensa se concentró en garantizar el reconocimiento de los títulos profesionales ya obtenidos, y su equiparación con los nuevos licenciados universitarios. La incertidumbre que en algún momento rodeó a esta equivalencia, generó el temor de que los profesionales no-universitarios fueran discriminados y considerados de segunda categoría.

- Práctica v/s teoría: de manera general, tanto en los antecedentes de contexto, como en las noticias analizadas, se puede notar la presencia de dos tradiciones en la enseñanza del periodismo. Por una parte, la centrada en la práctica como 
eje de la formación, modelo presente en los inicios de la enseñanza del periodismo en España. Por otra, aquella que da énfasis a la teoría y reflexión, entendiendo este elemento como uno de los ejes fundamentales del desarrollo científico del conocimiento. Lo anterior queda claro, por ejemplo, en el título de la última noticia publicada en 1971 sobre el tema cuando, y como punto final de la III Asamblea General de la Agrupación Nacional Sindical de Radio y TV, se concluyó que "Apoyaremos que el plan de estudios de Ciencias de la Información sea práctico y no de ciencia pura exclusivamente".

El texto, publicado en La Vanguardia, data del 19 de diciembre de 1971, pero da cuenta de una discusión que hoy, en pleno 2012, se mantiene vigente en las aulas del periodismo universitario.

\section{Bibliografia}

ANECA (2005) Libro blanco de los Títulos de Grado en Comunicación. Madrid: Agencia Nacional de Evaluación de la Calidad y Acreditación.

BARRERA, C. (2002). Notas para una historia del Instituto de Periodismo de la Universidad de Navarra (1958-1971). En Revista Comunicación y Sociedad, 2002, vol. XV, no 1. Navarra: Facultad de Comunicación, Universidad de Navarra. Pág.7-38. Disponible en: http://www.unav.es/fcom/comunicacionysociedad/es/articulo.php?art_id=336 [Visitado el 9-11-2012]

BENITO, A. (2002). Ciencia y enseñanza del periodismo. En Almuiña, Celso; Sotillos, Eduardo (coord.), Del Periódico a la Sociedad de la Información (II). 1era edición. Madrid: Sociedad Estatal España Nuevo Milenio. Pág. 301-311

GARCÍA JIMÉNEZ, L. (2007). Las teorías de la comunicación en España: un mapa sobre el territorio de nuestra investigación (1980-2006). Madrid: Editorial Tecnos (Grupo Anaya S.A.)

GORDON PÉREZ, M. (1991). La enseñanza del periodismo en el mundo occidental: estudio histórico de tres escuelas comparadas. Tesis Doctoral, Facultad de Ciencias de la Información, Departamento de Periodismo, Universidad Complutense de Madrid. Disponible en: http://eprints.ucm.es/1763/ [Visitado el 9-11-2012], Hemeroteca digital diario La Vanguardia. www.lavanguardia.org [Visitado el 15-10-2012], Hemeroteca digital diario ABC. www.abc.es [Visitado el 15-10-2012] 
MARTÍNEZ NICOLÁS, M. (2006). Masa (en situación) crítica. La investigación sobre periodismo en España: comunidad científica e intereses de conocimiento. En Anàlisi. Quaderns de Comunicació i Cultura, no 33. Barcelona: Universitat Autònoma de Barcelona: Facultat de Ciencies de la Comunicació. Pág. 135-170.

MARTÍNEZ NICOLÁS, M. (2009). La investigación sobre comunicación en España. Evolución histórica y retos actuales. En Revista Latina de Comunicación Social, no 64. La Laguna (Tenerife): Universidad de La Laguna. Pág. 1-14

MORAGAS, M. DE. (1991) Teorías de la Comunicación. Investigaciones sobre medios en América y Europa. 5ta edición (1era edición, 1981), Barcelona: Editorial Gustavo Gili S.A., GG MassMedia.

NOGUÉ, A.; Y BARRERA, C. (2006). La Vanguardia. Del franquismo a la democracia. Madrid: Editorial Fragua; colección Biblioteca de Ciencias de la Comunicación.

JONES, D. (1998). Investigación sobre comunicación en España: evolución y perspectivas. En Zer: Revista de estudios de comunicación, 5. Bizkaia: Facultad de Ciencias Sociales y de la Comunicación Universidad del País Vasco / Euskal Herriko Unibertsitatea. Disponible en http://www.ehu.es/zer/hemeroteca/pdfs/zer05-01-jones.pdf [Visitado el 9-122012]

OLMOS, V. (2002). Historia del ABC. 100 años clave en la historia de España. Barcelona: Plaza \& Janés Editores S.A.

PESTANO RODRÍGUEZ, J. M.; RODRÍGUEZ WANGÜEMERT, C.; DEL PONTI, P. (2011) "Transformaciones en los modelos de formación de periodistas en España. El reto europeo". En Estudios sobre el mensaje periodístico, vol. 17, no 2. Madrid: Universidad Complutense de Madrid. Pág. 401-415.

REAL, E. (2009). Los estudios de periodismo y el EEES: ¿Avance o retroceso?. En Icono 14. Revista de Comunicación y Nuevas Tecnologías, no14. Madrid: Icono 14, Asociación Científica, 2009. Pág. 08-34.

REGISTRO de Universidades, Centros y Títulos (RUCT), de la Secretaría de Estado de Educación, Formación Profesional y Universidades, del Ministerio de Educación, Cultura y Deporte de España. https://www.educacion.gob.es/ruct/home [Visitado el 8-12-2012]

VIGIL Y VÁZQUEZ, M. (1987) El periodismo enseñado. De la Escuela de "El Debate" a Ciencias de la Información. 1era ed. Barcelona, Editorial Mitre, Colección Ciencias de la Comunicación. 